\title{
The Influence of Binary Stars on Post-Collapse Evolution
}

\author{
Rosemary Apple \\ School of Mathematics, University of Edinburgh, UK \\ email: r.apple@sms.ed.ac.uk
}

\begin{abstract}
The results in the $N$-body simulations in Giersz \& Heggie (1996) show that although the masses segregate as expected during core collapse, after core collapse there is self-similar evolution with very little further evidence of mass segregation even though the system has not reached equipartition. Binary stars halt core collapse and it is possible that they also halt the tendency toward equipartition. To investigate this problem, we construct two models. One model is a two-component model which assumes that binary stars form in the region dominated by heavy stars. The other model is a single mass model which assumes that binary stars form only in the region of the core. In both models, when the binary heating term is included, we find the post-collapse evolution to be self-similar. The aim of our work is to combine these two models to form a two-component model which assumes that binary formation only occurs in the core.
\end{abstract}

Keywords. binaries: general, stellar dynamics, methods: analytical

\section{Two-Component Model}

In our first model, we consider a two-component system with individual masses $m_{2}>$ $m_{1}$. The total mass of the heavy stars and the total mass of the light stars are $M_{2}$ and $M_{1}$ respectively. We build our two-component model from equation (1) in Spitzer (1969). We assume that the heavy stars are uniformly distributed in the sphere characterized by the half-mass radius of the heavy stars, $r_{2}$. Similar assumptions are made with the light stars, where the half-mass radius is $r_{1}$. We use the binary heating term derived in Heggie \& Hut (2003) and assume that binary stars form throughout the region of heavy stars. From these assumptions, we derive the equations for our dynamical system,

$$
\begin{aligned}
& \dot{r}_{1}\left(r_{1}, r_{2}\right)=f\left(r_{1}, r_{2}\right) \frac{\phi\left(\frac{r_{2}}{r_{1}}\right)}{t_{e q}\left(r_{1}, r_{2}\right)}, \\
& \dot{r}_{2}\left(r_{1}, r_{2}\right)=\frac{g\left(r_{1}, r_{2}\right) f\left(r_{1}, r_{2}\right) \phi\left(\frac{r_{2}}{r_{1}}\right)}{t_{e q}\left(r_{1}, r_{2}\right)}+\frac{9.688 G^{1 / 2} M_{2}^{2} m_{2}^{3} r_{1}^{27 / 2} r_{2}^{-1 / 2}}{\left(M_{2} r_{1}^{3}+M_{1} r_{2}^{3}\right)^{7 / 2}\left(M_{2} r_{1}^{3}+2 M_{1} r_{2}^{3}\right)},
\end{aligned}
$$

where, $f\left(r_{1}, r_{2}\right)=\left[r_{1}^{4} r_{2} M_{1}^{2} M_{2}\left(M_{1}+M_{2}\right)\right]^{-1}, g\left(r_{1}, r_{2}\right)=\frac{M_{1} r_{2}^{2}\left(3 M_{2} r_{2}^{2}-M_{1} r_{1}^{2}-M_{2} r_{1}^{2}\right)}{M_{2} r_{1}\left(M_{2} r_{1}^{3}+2 M_{1} r_{2}^{3}\right)}$,

$$
\begin{gathered}
\phi\left(\frac{r_{2}}{r_{1}}\right)=\frac{M_{2}}{M_{1}} \frac{m_{2}}{m_{1}}+\frac{m_{2}}{m_{1}}\left(\frac{r_{2}}{r_{1}}\right)^{3}-\left(1+\frac{M_{2}}{M_{1}}\right) \frac{r_{2}}{r_{1}}, \text { and } \\
t_{e q}\left(r_{1}, r_{2}\right)=\frac{\sqrt{\pi}\left(\left(M_{1}+M_{2}\right) r_{1}^{2} r_{2}+M_{2} r_{1}^{3}+M_{1} r_{2}^{3}\right)^{3 / 2}}{6 \sqrt{6} M_{1} m_{2} \log \left(M_{1} / m_{1}\right)\left(r_{1} r_{2}\right)^{3 / 2}} .
\end{gathered}
$$

The second term on the right in Eqn. (1.2) is due to binary heating.

In Fig. 1(i) \& (ii), we show the phase portraits for $r_{2}$ vs. $r_{1}$. We use an example in which the system is Spitzer stable, where $m_{2} / m_{1}=2$ and $M_{2} / M_{1}=0.1$. In the case where binary heating is excluded, we find two lines of equilibria (depicted by the dashed 

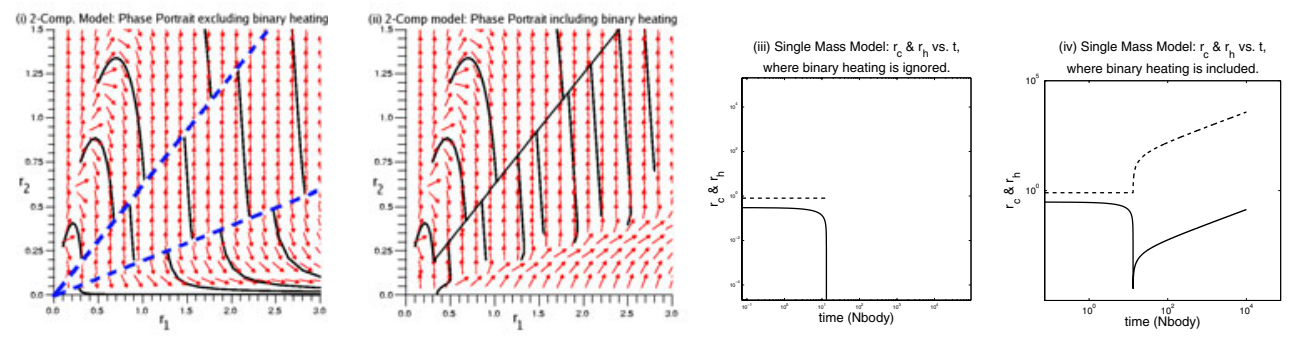

Figure 1. From left to right: Phase portraits for the two-component model (i) ignoring binary heating and (ii) including binary heating and the graphs of $r_{h}$ (dashed) and $r_{c}$ (solid) vs. $t$ in the single mass model (iii) ignoring binary heating and (iv) including binary heating.

lines in Fig. 1(i)). The upper line is stable and the lower line is unstable. When we include binary stars, the system reaches what was the stable line of equilibria in the case without binary stars. When this line is reached, the system is in quasi-equilibrium.

\section{Single Mass Model}

The second model is a single mass model which assumes that binary stars only form in the core of the cluster. To model this system, we use approximations based on LyndenBell \& Eggleton (1980) and again the binary heating term found in Heggie \& Hut (2003). From this, we get the equations for our model

$$
\begin{aligned}
& \dot{r}_{h}=\frac{765}{64 \pi^{2}} G^{\frac{1}{2}} N^{-\frac{5}{2}} m^{\frac{1}{2}} r_{c}^{-4+\frac{1}{2} \alpha} r_{h}^{\frac{7}{2}-\frac{1}{2} \alpha}, \\
& \dot{r}_{c}=\frac{1}{5-2 \alpha}\left[-\frac{r_{c}}{t_{r c}}+\frac{765}{16 \pi^{2}} G^{\frac{1}{2}} N^{-\frac{5}{2}} m^{\frac{1}{2}} r_{c}^{-8+\frac{5}{2} \alpha} r_{h}^{\frac{15}{2}-\frac{5}{2} \alpha}+(6-2 \alpha) \frac{r_{c}}{r_{h}} \dot{r_{h}}\right],
\end{aligned}
$$

where, $t_{r c}=G^{\frac{3}{2}} N^{\frac{1}{2}} m^{-\frac{1}{2}} r_{c}^{3-\frac{1}{2} a} r_{h}^{\frac{-3}{2}+\frac{1}{2} a}\left(3 \log \left(.5 N\left(r_{c} / r_{h}\right)^{3-a}\right)\right)^{-1}$.

In this model, all but the first term in equation (2.2) are caused by binary heating.

The evolution of the half-mass radius and the core radius over time is shown in Fig. 1(iii) \& (iv). As expected, the core collapses when the binary heating term is ignored. When binary heating is included, the core radius collapses to a minimum value and then the post-collapse evolution is self-similar.

\section{Further Work}

We intend to combine these two models to create a new model which will include mass segregation and will assume that binary stars exist only in the core. From this model we can determine whether the influence of binary stars causes the post-collapse evolution to be self-similar.

\section{References}

Giersz, M. \& Heggie, D. C. 1996, MNRAS 279, 1037

Heggie, D. C. \& Hut, P. 2003, Gravitational Million Body Problem (Cambridge University Press, Cambridge)

Lynden-Bell, D. \& Eggleton, P. P. 1980, MNRAS 483, 498

Spitzer, L. Jr. 1969, ApJ 158, 139 